\title{
Far Infrared Optical Absorption Study and Characterization of $\mathrm{Y}_{1} \mathrm{Ba}_{2} \mathrm{Cu}_{3} \mathrm{O}_{7-\delta} / \mathrm{Pr}_{0.5} \mathrm{Y}_{0.5} \mathrm{Ba}_{2} \mathrm{Cu}_{3} \mathrm{O}_{7-\delta}$ Superlattices
}

\author{
T. R. Yang", H. C. Yang', H. E. Horng" and Y. K. Chyan" \\ Department of Physics, National Taiwan Normal University, Taipei 117, Taiwan \\ bepartment of Physics, National Taiwan University, Taipei, Taiwan
}

We report infrared reflection measurements on $\mathrm{Y}_{1} \mathrm{Ba}_{2} \mathrm{Cu}_{3} \mathrm{O}_{7.8} / \mathrm{Pr}_{0.5} \mathrm{Y}_{0.5} \mathrm{Ba}_{2} \mathrm{Cu}_{3} \mathrm{O}_{7-8}(60 \mathrm{~A} / 60 \AA$, 16 layers and $60 \AA / 24 \AA, 16$ layers on $\mathrm{SrTiO}_{3}(100)$ substrate) high $\mathrm{T}_{\mathrm{C}}$ superconducting superlattice films. We measured absorption spectrum of these films from $30-700 \mathrm{~cm}^{-1}(3.7-66.9 \mathrm{meV})$ and $80-300 \mathrm{~K}$. These films showed resistive superconducting transitions with an on set at $80 \mathrm{~K}$. Some of the phonons are smear out because of strong electron-phonon mediation. The appearance of vibration modes are around 547 、361 、279 、172 . $150 、 125 \mathrm{~cm}^{-1}$ at $80 \mathrm{~K}$. Some contribution and interaction between layers were observed from measured spectra. The different configuration of interlayer thickness of these superlattices have $1-2 \mathrm{~cm}^{-1}$ peaks shift in spectra..The dielectric and electron-phonon behaviors due to interaction of superlattice layers will go further studies.

(This works supported by National Science Consul: NSC 86-2112-M-003-005).

\section{INTRODUCTION}

Scientists pay a lot love of attention to the high $T_{c}$ superlattices after the first superlattice YBCO/DBCO had been successfully made by Triscone at al.[1] More recently, several groups have reported the superconducting properties of alloys of $\mathrm{Y}_{1} \mathrm{Ba}_{2} \mathrm{Cu}_{3} \mathrm{O}_{7-8} / \mathrm{Pr}_{0.5} \mathrm{Y}_{0.5} \mathrm{Ba}_{2} \mathrm{Cu}_{3} \mathrm{O}_{7}$ layered structure superconductors.[2-6] This material has been extensively studied. [7] The coupling and layer number of copper oxide layer in high temperature sperconductor has play an important roles for the superconductivity.[8] In this paper we study the dielectric mechanism of these superconducting layers of multilayer high $T_{c}$ superlattice films. [9-14]

It is interesting to know the infrared (IR) behaviors of these films and its dielectric behavior of different layer configuration. In this article, we report the detailed temperature dependent infrared absorption study of YBCO/PYBCO superlattice over the temperatures between 80 and $300 \mathrm{~K}$. The phonon behavior of these materials were studied and discussed.

\section{EXPERIMTNT}

0921-4534/97/\$17.00 Elsevier Science B.V. All rights reserved PII S0921-4534(97)00600-X
$\mathrm{Y}_{1} \mathrm{Ba}_{2} \mathrm{Cu}_{3} \mathrm{O}_{5} / \mathrm{Pr}_{0.5} \mathrm{Y}_{0.5} \mathrm{Ba}_{2} \mathrm{Cu}_{3} \mathrm{O}_{7}$ (YBCO/PYBCO, 6OA $60 \AA 16$ layers and $60 \AA 24 \AA 16$ layers on (100) $\mathrm{SrTiO}_{3}$ substrate) high $\mathrm{T}_{C}$ superconducting superlattice films were prepared by radio frequency magnetron sputtering (RF sputtering).[15] The configuratuion of RF sputtering system is shown in Fig. 1. A commencial type $5 \times 5 \times 0.5 \mathrm{~mm}^{3}, 99.95 \%$ pure single crystal $\mathrm{SrTiO}_{3}$ with mirror like surface in (100) face were used as substrate. These film showed resistive superconducting transitions with an on set at $80 \mathrm{~K}$ by resistivity vs temperature measurements. These samples also examined by powder X-ray

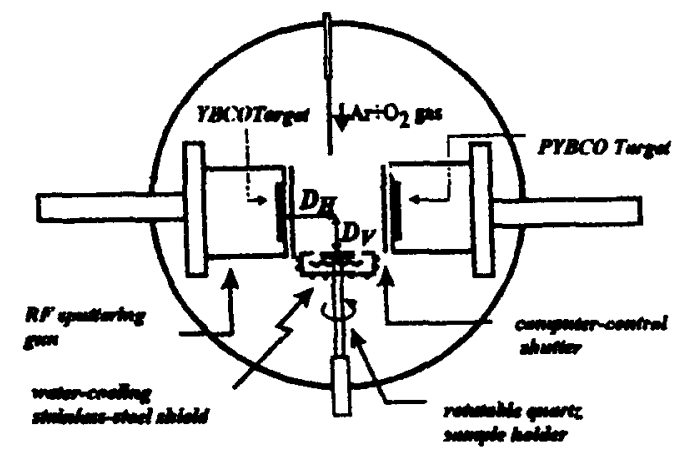

Fig. 1. The configuration diagram of RF sputtering system for these samples 
diffraction, SQUID - Hall measurement. All of these examinations and measurements ensured that these superlattice films have good quality.

Infrared absorption measurements of reflectivity at near normal incident were made on Bruker IFS$120 \mathrm{HR}$ with a resolution of $2 \mathrm{~cm}^{-1}$ ranging from 30 $700 \mathrm{~cm}^{-1}$. Mecury-Arc lamp was used for far-infrared source. High throughput mylar beamspliter was used in our experiments. The radiation was detected by a Liquid-helium-cooled silicon bolometer (Noise equivalent power $=1.3 \times 10^{-13}$ W/Hz ${ }^{0.5}$ ) with suitable filters. The specimens were mounted on the liquidnitrogen finger tip type continuous flow APD cryogenics with $x-y-z$ sample stage. A mirror like gold was mounted next by the sample on the cold finger tip of cryogenics. The absolute reflectivity value of samples were done by comparision with the gold mirror. The measurements of samples and gold mirror were made at temperature between 80 to $300 \mathrm{~K}$. The temperature of finger tip inside cryogenics were controlled by Lake-shore 331 temperature controller with a temperature stability of $0.5 \mathrm{~K}$ and better.

\section{RESULTS AND DISCUSSIONSS}

The infrared reflection measurements on YBCO/PYBCO (60A60A, 16 layers) is shown in Fig.

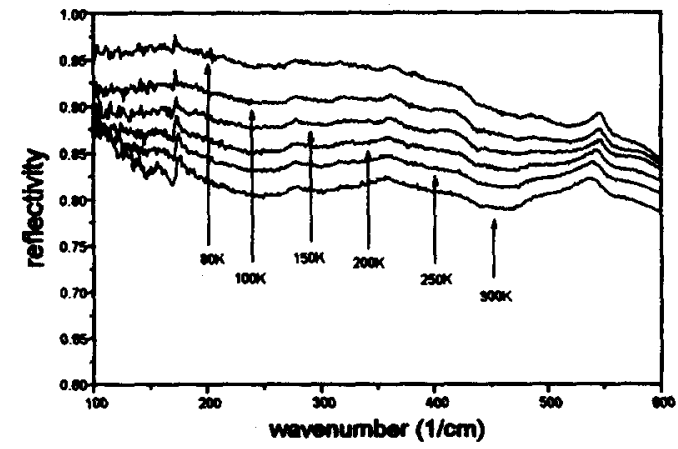

Fig. 2. Infrared reflection measurements of YBCO/PBCO (60A/60A, 16 layers) supertattice

2. The appearance of vibration modes are around $547 、 361,279 、 172 、 150 、 125 \mathrm{~cm}^{-1}$ at $80 \mathrm{~K}$. Some contribution and interaction between layers were observed from measured spectra. The infrared reflection measurements on YBCO/PYBCO $(60 \AA 24 \AA, 16$ layers) superlattice films is shown in Fig. 3. Some of the phonons are smear out because of strong electron-phonon mediation. The different configuration of interlayer thickness of these superlattices have $1-2 \mathrm{~cm}^{-1}$ peaks shift in spectra.

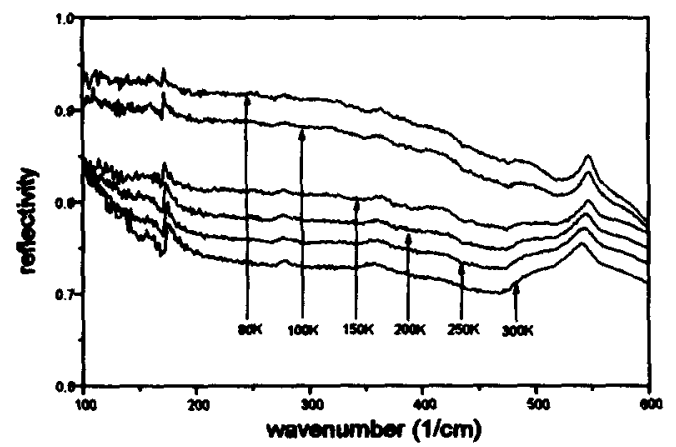

Fig. 3. Infrared reflection measurements of YBCO/PBCO (60A/24A, 16 layers) superlattice

In normal state, we fit this spectrum with assuming Drude-like free carriers as well as lorentzian phonon ascillators of films dielectric function. In superconducting state, the contribution of conductivity was added to dielectric function. The electron-phonon behaviors due to interaction of superiattice layers, carrier concentration, mobility, effective mass as well as their dielectric behavior of these crystals will go further study.

\section{REFLRENCES}

1. C. T. Roger et al. Appl. Phys. Lett 55, 2032 (1989)

2. U. Poppe et al., Solid State Commun. 71, 569 (1989)

3. J. M. Triscone et al., Phys. Rev. Lett 64, 804 (1990)

4. D. H Lowndes et al., Phys. Rev. Lett. 65, 1060 (1990)

5. Q. Li, et al.,Phys. Rev. Lett. 64, 3086 (1990)

6. J.H Chen Thesis (1995)

7. J. J. Neumeier et al., Phys. Rev. Lett 63, 2516 (1989)

8. R. F. Wood et al., Physica C 165, 97 (1990)

9. J. Pennycook et al., Phys. Rev. Lett 67, 765 (1991)

10. C. L. Jia et al., Physica C 210, 1 (1993)

11. H. R. Kenchner et al., Physica C 198, 75 (1992)

12. M. Affronte et al., Phys. Rev. B. 43, 11484 (1991)

13. R. F. Wood et al., Phys. Rev. Lett 66, 829 (1991)

14. M. Rasolt et al., Phys Rev. Lett 66, 2927 (1991)

15. L. M Wang $P h$. D. disserataion, National Taiwan Normal University, Taipei Taiwan, P6 (1994) 